\title{
Erratum: Modeling the long-term evolution of dilute solid solutions in the presence of vacancy fluxes [Phys. Rev. Materials 2, 073605 (2018)]
}

\author{
Thomas Schuler, Pascal Bellon, Dallas R. Trinkle, and Robert S. Averback
}

(Received 20 August 2019; published 2 October 2019)

DOI: 10.1103/PhysRevMaterials.3.109901

In the Appendix, we noted some errors in some of the analytical expressions for transport coefficients. These mistakes do not impact any conclusion of the paper but prevent readers from using the analytical expressions to reproduce our results and/or use them in other systems. We only provide the correction for erroneous expressions, namely, Table I and Eqs. (A11) and (A12) in the paper.

\section{CORRECTIONS TO APPENDIX A}

$$
\begin{gathered}
L_{V V}=L_{V V}^{V}\left\{1+\kappa\left[W_{2}\left(1035+W_{1} P_{0}+W_{3} P_{3}\right)+W_{1} P_{4}+W_{3} P_{5}\right]\right\}, \\
\kappa=\frac{[B]}{\left(W_{1}+W_{2}\right) P_{0}+W_{3} P_{2}} .
\end{gathered}
$$

The authors thank C. Daniels for pointing out the errors.

TABLE I. Coefficients of polynomial functions obtained for a correlation radius $R_{\text {cor }}=4 a$. These coefficients are obtained from selfconsistent mean field calculations.

\begin{tabular}{lccccc}
\hline \hline$\alpha_{j, k}$ & $k=0$ & $k=1$ & $k=2$ & $k=3$ & $k=4$ \\
\hline$j=0$ & +1.0000 & +1.3508 & +0.56862 & +0.089534 & +0.0044380 \\
$j=1$ & -9.4832 & -6.3630 & -1.0109 & -0.0030132 & +0.0044380 \\
$j=2$ & +3.5000 & +3.2364 & +0.96152 & +0.11299 & +0.0044380 \\
$j=3$ & +1443.5 & +621.79 & +99.670 & +5.0537 & +0.0044380 \\
$j=4$ & +1035.0 & +1466.0 & +637.76 & +102.65 & +5.1727 \\
$j=5$ & +3622.6 & +3418.5 & +1044.8 & +126.95 & +5.1727 \\
\hline
\end{tabular}

